\title{
LY294002 Inhibit Proliferation, Migration, and Invasion of Craniopharyngiomas via PI3K/Akt Signaling Pathway
}

\section{Lin Zhou}

First Affiliated Hospital of Nanchang University

\section{Cheng Xing Yang}

First Affiliated Hospital of Nanchang University

\section{Lin Chun Fang}

First Affiliated Hospital of Nanchang University

\section{You Yuan Bao}

First Affiliated Hospital of Nanchang University

\section{Zhi Gang Wang}

Shanghai 9th Peoples Hospital Affiliated to Shanghai Jiaotong University School of Medicine

\section{Shao Yang Li}

First Affiliated Hospital of Nanchang University

\section{Min De Li}

First Affiliated Hospital of Nanchang University

Shi Hai Lan

First Affiliated Hospital of Nanchang University

\section{Xiao Wu}

First Affiliated

\section{Zhi Gao Tong}

First Affiliated Hospital of Nanchang University

\section{Su Yue Zheng}

First Affiliated Hospital of Nanchang University

\section{Bin Tang}

First Affiliated Hospital of Nanchang University

\section{Tao Hong ( $\sim$ ht2000@vip.sina.com )}

First Affiliated Hospital of Nanchang University https://orcid.org/0000-0002-2693-8991

\section{Research}

Keywords: LY294002, craniopharyngiomas, PI3K/Akt signaling pathway 
Posted Date: October 28th, 2020

DOI: https://doi.org/10.21203/rs.3.rs-96933/v1

License: (c) (i) This work is licensed under a Creative Commons Attribution 4.0 International License. Read Full License 


\section{Abstract}

Objective

Craniopharyngiomas are rare histologically benign but clinically challenging neoplasms. The aim of this study is to explore the effect and significance of PI3K signal pathway on papillary craniopharyngioma cell growth and survival.

Methods

Western blotting (WB) was used to evaluate expression of the PI3K / AKT protein in Craniopharyngiomas tissues relative to health controls. Primary tumor cells were obtained from fresh papillary craniopharyngioma samples by primary cell culture and determined by cell morphology, immunofluorescence staining and specific cell markers expression. In this study, PCPs cell lines, isolated from fresh papillary craniopharyngioma samples, were treated with different concentrations of LY294002, a specific inhibitor of the PI3K / AKT signaling pathway, in order to evaluate their proliferation, migration and invasion. The proliferation effects was determined using Cell Counting Kit-8 and colony formation. Cell apoptosis and cell cycle were detected by flow cytometry. Furthermore, cell migration and invasion levels were detected by wound healing and Transwell assays, respectively.

Results

The expression of p-PI3K and p-AKT were obviously higher in human craniopharyngioma tissue relative to their corresponding normal control. PCPs primary cells were isolated and detected by immunofluorescence, and PCPs cytokeratin (pan CK) and vimentin protein (VIM) were detected.The inhibition of PI3K / AKT signaling pathway can significantly inhibit the proliferation, migration and invasion of PCPs primary cells.

Conclusions

LY294002 effectively inhibits the proliferation, migration and invasion of PCPs cells through the PI3K / AKT signaling pathway.

\section{Introduction}

Craniopharyngiomas are rare malformational tumours of low histological malignancy arising along the craniopharyngeal duct. The two histological subtypes, adamantinomatous craniopharyngioma (ACP) and papillary craniopharyngioma (PCP), differ in genesis and age distribution. ACPs are diagnosed with a bimodal peak of incidence (5-15 years and 45-60 years), whereas PCPs are usually found in adults[1].

ACP tumorigenesis are characterized as activated Wnt signaling pathway, and nuclear b-catenin accumulation caused by CTNNB1 gene mutation,such mutations are reported in $16 \%-100 \%$ of ACP patients [2, 3].Tobias Goschzik et al.Report that the CTNNB1 mutations are exclusively found in exon 3 
encoding the degradation-targeting box where normally casein kinase 1 first phosphorylates Ser 45 . The mutation casuses b-catenin phosphorylation that affect the function of tumor suppression.In contrast, BRAF V600E mutations were detected in $81-100 \%$ of PCP,which constitutively activates the MEK/ERK pathway[3, 4].

During tumorigenesis, tumor cells use normal cell-mediated signaling pathways to achieve their own cell proliferation, migration, and anti-apoptosis, thereby gaining growth advantages over normal cells. Studies have found that some of these signaling pathways are mediated by cell membrane receptors [5]. At present, the two major types of membrane receptors studied are $G$ protein coupled receptors (GPCRs) and receptor tyrosine kinases (RTKs). PI3K is the main downstream effector of GPCRs and RTKs. Membrane receptors mediate PI3K activation and produce a second messenger phosphatidyl-3,4,5-triphosphate (PIP3) on the plasma membrane. PIP3 and the cell contain PH The signal protein Akt in the domain binds to phosphoinositide dependent kinase-1 (PDK1). Akt translocates to the cell membrane and obtains catalytic activity. It catalyzes its own Ser124 and Thr450 phosphorylation. PDK1 can catalyze Akt Thr308 phosphorylation; Akt may also activate Akt via phosphorylation of Ser473 via phosphinositide dependent kinase-2 (PDK2) (such as integrin-linked kinase ILK)[6]. Akt activated by PI3K can activate its downstream target protein mTOR through phosphorylation, forming mTORC1 complex and mTORC2 complex[7, 8]. mTORC1 promotes protein synthesis and maintains cell metabolism and cell growth by regulating p70S6K1 and 4EBP1; mTORC2 complex can activate Akt to inhibit the hydrolysis of cyclin D1 / E, thereby maintaining tumor cell proliferation. A number of studies have indicated that PTEN is able to dephosphorylate PIP3, antagonize PI3K activity and reduce the concentration of PIP3 within the cells, thereby inhibiting the activation of Akt, through which PTEN regulates cell apoptosis [7]. In addition, continuously activated Akt can not only prevented PTEN-mediated apoptosis, but also inhibit its apoptosis by phosphorylation of its downstream target proteins Bad, Caspase9, NF-kB, FOXP4, P21, etc, survival of tumor cells[7, 9]. Observed significantly increased expression of phosphorylated-PI3K(p-PI3K) and phosphorylated-AKT(p-AKT), the activated form of PI3K and AKT in craniopharyngiomas tissues. The proliferation and migration ability of PCPs cells treated with PI3K inhibitor LY294002 were significantly weakened, suggesting that the proliferation, migration and anti-apoptosis of craniopharyngioma cells are related to the activation of PI3K-Akt signaling pathway.

\section{Methods}

\section{Patients and samples collection}

Craniopharyngiomas tissue samples and healthy brain tissue samples were collected at Nanchang University (Nanchang China) First Affiliated Hospital by surgery. PCPs specimens were obtained from patients who had been pathologically and clinically diagnosed with PCPs. The tumours were either kept at $-80^{\circ} \mathrm{C}$ until DNA/protein extraction or collected immediately for primary culture after resection. Healthy brain tissue samples were obtained from patients with conditions other than CP such as acute brain trauma or epilepsy[10]. Written informed consent was obtained from all participants involved in the study 
before the clinical samples were used for research purposes. Ethical clearance was approved by the Research Ethical Committee of Nanchang University.

\section{Primary cell culture}

Fresh PCPs tissues were obtained from adult native mainly solid tumor specimens that had been directly captured after surgical removal. Only tissue including portions of tumor, as verified by instantaneous sections and microscopical inspection, was further used. After washing with phosphate-buffered saline (PBS) containing 1\% penicillin/streptomycin (Life Technologies, Gibco BRL, Grand Island, USA), the specimens were dissected into pieces 2 to $3 \mathrm{~mm} 3$ in size and digested with $0.25 \%$ trypsin (Sigma-Aldrich, St. Louis, MO, USA) and $1 \mathrm{mg} / \mathrm{mL}$ collagenase II (Sigma-Aldrich, St. Louis, MO, USA) for 30 minutes at $37^{\circ} \mathrm{C}$ in an incubated shaker. After centrifugation at $1000 \times \mathrm{g}$ at $4{ }^{\circ} \mathrm{C}$ for 10 minutes, the supernatant was discarded, and the pellet was resuspended with Dulbecco's modified Eagle's medium (high glucose, DMEM-HG) with $10 \%$ fetal bovine serum (Life Technologies). Then, suspended primary PCPs cells were cultured in defined serum-free keratinocyte medium (Invitrogen, Carlsbad, CA, USA). A constanttemperature incubator maintained at $37^{\circ} \mathrm{C}$ was used to incubate cells in $5 \% \mathrm{CO}_{2}$. Cell purification was carried out to establish a well-formed primary PCPs cell line.

\section{Cell counting kit-8 assay}

$4 \times 10^{3}$ cells in suspension were seeded in a 96-well plate. Different concentrations of LY294002 were adopted to treat cells for $4 \mathrm{~h}$ with three replicates. Then, $10 \mu \mathrm{l} \mathrm{CCK}-8$ reagent was added to the well for $4 \mathrm{~h}$ to form formazan. At last, the plate was read at $450-490 \mathrm{~nm}$ on a microplate reader.Six replicate wells were set up in each group.

\section{Colony formation assay}

A total of $5 \times 10^{2} /$ well cells were firstly seeded in 6-well plates until most of them were attached to the bottom. Then, different concentrations of LY294002 was used for 6-well plates. After two weeks, the cells were fixed and stained. Colonies containing $\geq 20$ cells were counted.

\section{Wound healing assay}

A total of $5 \times 10^{4}$ cells was initially seeded in each well of a 6 -well plate for 24 hours. Cell layer was scratched by a pipette tip $(200 \mu \mathrm{L})$. Subsequently, cells were then continued cultured with indicated concentrations of LY294002. Representative wound was imaged by an optical microscope. Each experiment was conducted for three times.

\section{Cell invasion and migration assay}

The Transwell cell migration and invasion assay was employed to assess cell migratory and invasive abilities. Cells were seed into 24-well Transwell Boyden chambers ( $8.0 \mu \mathrm{m}$ pore size; Costar, Cambridge, MA) based on the manufacturer's instructions. Briefly, for the Transwell cell migration assay, $5 \times 10^{4}$ cells were suspended in $200 \mu \mathrm{l}$ DMEM without FBS and added to the upper chambers, then $700 \mu$ DMEM containing $30 \%$ FBS was added into the lower chambers and incubated for $24 \mathrm{~h}$ at $37^{\circ} \mathrm{C}$. For the 
Transwell cell invasion assay, $5 \times 10^{4}$ cells were suspended in $200 \mu$ DMEM without FBS and added to the upper chambers, which had been filled with $100 \mu$ l Matrigel, then $700 \mu l$ DMEM containing 30\% FBS was added into the lower chambers and incubated for $48 \mathrm{~h}$ at $37^{\circ} \mathrm{C}$. Thereafter, non-invasive cells on the inner surfaces of the upper chambers were gently scraped off using a cotton swab. Invasive cells were fixed with $100 \%$ methanol, stained with $0.5 \%$ crystal violet solution, and washed with $1 \times$ PBS. Following these procedures, the cells were counted and photographed using a microscope $(\times 100)$ with at least three independent experiments.

\section{Apoptosis analysis}

Apoptotic cell death was measured using AnnexinV-APC/7-AAD, according to the manufacturer's protocols. Briefly, PCPs cancer cells were seeded at a densities of $5 \times 10^{4}$ cells per well in 6 -well plates, then cultured for $24 \mathrm{~h}$ in DMEM medium. Samples were subsequently treated with various concentrations of LY294002 $(0,10,20,30,60$ and $90 \mu \mathrm{M})$ for $24 \mathrm{~h}$, after which cells were digested with $0.25 \%$ pancreatin without EDTA and washed twice with $0.001 \%$ FBS-PBS buffer. Next, $100 \mu \mathrm{L}$ of reagent was added to micro-centrifuge tubes, after which $10 \mu \mathrm{L}$ of cell suspension was added to each tube and incubated for $10 \mathrm{~min}$ at room temperature. Finally, the cells were analyzed using a BriCyte E6. Flow cytometry data was obtained from 5000 events (gated cells) per sample and the percentages of cells shown in the figures were calculated from the mean fluorescence intensity in each of the four quadrants. The coefficient of variation from the mean fluorescence was less than $10 \%$.

\section{Cell cycle analysis}

Cell cycle assays was measured using cell cycle analysis kit (KGA512)according to the protocols. PCPs cancer cells were seeded at a densities of $5 \times 10^{4}$ cells per well in 6-well plates, then cultured for $24 \mathrm{~h}$ in DMEM medium. Samples were subsequently treated with various concentrations of $\operatorname{LY} 294002(0,10$, $20,30,60$ and $90 \mu \mathrm{M}$ ) for $24 \mathrm{~h}$, after which cells were digested with $0.25 \%$ pancreatin without EDTA and washed twice with $0.001 \%$ FBS-PBS buffer. Resuspend the cells in 100 ul PBS and slowly add 700 ul prechilled $80 \%$ ethanol to make the final ethanol concentration $70 \%$, at $4{ }^{\circ} \mathrm{C}$ fixed for $4 \mathrm{~h}, 1000 \mathrm{rpm}, 5 \mathrm{~min}$, pre-cold PBS rinse twice, add $100 \mathrm{ul} \mathrm{RNase} \mathrm{(50ug} \mathrm{/} \mathrm{ml),} 37^{\circ} \mathrm{C}$ water bath for $30 \mathrm{~min}$.Next add $400 \mathrm{ul} \mathrm{PI}$ (50ug / $\mathrm{ml}$ ) and stain at $4{ }^{\circ} \mathrm{C}$ for $30 \mathrm{~min}$ in the dark. Finally, the cells were analyzed using a BriCyte E6.

\section{Immunofluorescence assay}

Primary PCPs cells were washed with PBS and then fixed in 75\% ethanol for 20 minutes at room temperature. Following washing with PBS, the cells were blocked with $5 \%$ bovine serum albumin (BSA) for $1 \mathrm{~h}$ at $37^{\circ} \mathrm{C}$. The cells were then incubated with monoclonal anti-human pan-cytokeratin (pan-CK, Abcam, ab215838, 1:100, Cambridge, UK), anti-vimentin (VIM, CST, 5742T, 1:100), anti- p-PI3K (p85) (Abcam, ab182651, 1:200), anti-PI3K (Abcam, ab191606, 1:200), anti-p-AKT (S473) (Abcam, ab8932, 1:200), and anti-AKT (Abcam, ab8805, 1:200) overnight at $4{ }^{\circ} \mathrm{C}$. The secondary antibody was Alexa 488conjugated goat anti-mouse or anti-rabbit IgG (Abcam). To counterstain nuclei,4',6-diamidino-2phenylindole (DAPI, $10 \mu \mathrm{g} / \mathrm{mL}$, 32670; Sigma-Aldrich) was used. Fluorescent images were obtained using a microscope (Carl Zeiss, Germany) and processed using Photoshop software (Adobe). 


\section{Western blotting (WB)}

Total proteins were extracted from the cultured cells after treatment with or without LY294002 and PCPs tissue were prepared using RIPA lysis buffer (Beyotime Institute of Biotechnology, Jiangsu, china). Proteins were quantified using a Pierce BCA protein Assay kit (Thermo Fisher Scientific, Inc.). Cell or tissue lysates of 30 ug protein samples were then separated by SDS-PAGE on 10-15\% gels and transferred to PVDF membranes (Millipore, Billerica, MA, USA). The membranes were then stained with $0.5 \%$ Ponceau $S$ with $1 \%$ acetic acid to ensure equal loading. After blocking in PBS with Tween-20 (PBS$\mathrm{T}$ ) with $5 \% \mathrm{BSA}$ for $1 \mathrm{~h}$, the PVDF membranes were incubated overnight with the following primary antibodies: monoclonal antibodies against GAPDH (Abcam, ab8245, 1:1000), p-PI3K (p85) (Abcam, ab182651, 1:5000), PI3K (Abcam, ab191606, 1:5000), p-AKT (S473) (Abcam, ab8932, 1:2000), and AKT (Abcam, ab8805, 1:2000). The membranes were then washed with Tris-buffered saline with Tween-20 (TBST) (10 minutes, three times) and incubated with the secondary antibody horseradish peroxidase (HRP)-labeled goat anti-rabbit (CST, 7074, 1:4000) or anti-mouse (CST, 7076, 1:4000) for $1 \mathrm{~h}$ at room temperature (RT). Protein bands were visualized by enhanced chemiluminescence (ECL).

\section{Statistical analysis}

All data are presented as mean \pm SEM. All experiments are performed at least in three independent times. By the means of one-way analysis of variance followed by the Duncan multiple-comparison test using SPSS 19.0 (SPSS Inc., Chicago, IL), we calculate the statistical significance. $P<0.05, P<0.01$, or $P<0.001$ is regarded as statistically significant.

\section{Results}

\section{P-PI3Kワp-AKT expression level in PCPs cancer tissues.}

To study whether the PI3K/AKT signaling pathway is clinically relevant in PCPs cancer, the expression levels of p-PI3K and total PI3K,p-AKT and total AKT were determined in human PCPs tissue by Western blot (WB), using normal brain tissue as a control group[10]. Each group performed at least three biological replicates. Compared with the normal brain tissues, higher expression level of $\mathrm{p}-\mathrm{PI} 3 \mathrm{~K} /$ total PI3K ratio $\square$ p-AKT/total AKT ratio was observed in the PCPs tissues according to Western blot (WB) analysis (P $<0.001$ ) (Fig. 1). Moreover,p-PI3K》 p-Akt protein expression levels in PCPs cells treated with LY294002 were correspondingly decreased compared with untreated(Fig. 3). The present findings indicated that LY294002 effectively suppressed the activation of the PI3K/Akt pathway.

\section{Successful cultivation of PCPs primary tumor cells.}

To further determine the biology functions of PI3K/AKT in PCPs cells. We successfully isolated and cultured primary PCPs cells from the $2 \#$ patient for the functional assay in vitro subsequent experiments. Isolated primary cells were cultured for 7 days, cell morphology was observed by inverted contrast microscope directly. Results showed that the primary cells had prominent spindle-like, round, or triangle 
cell bodies just as "paving stone". In contrast, as known, keratins was the molecular markers for tumors with an epithelial origin[11, 12], and vimentin (VIM) is effective for tumor diagnosis in surgical pathology for epithelial tumor cells cannot react with anti-vimentin (anti-VIM)[13]. Therefore, we detect the pan-CK and VIM as the positive and negative control of primary PCPs cell by the means of immunofluorescence. Results showed that pan-CK expressed in the cytoplasm of PCPs cell and identified as positive (down), while VIM cannot be detected and identified as negative (up) (Fig. 2). These results indicated that the primary cell model was PCPs cells and not contaminated by craniopharyngia-associated fibroblasts.

\section{Effects of the PI3K inhibitor LY294002 on regulation of PCPs cell proliferation.}

The inhibitory effect of LY294002 on the viability of PCPs cell lines was examined using an CCK assay. The results revealed that PCPs cell lines were sensitive to LY294002 and LY294002 inhibits PCPs cell viability in a time and dose-dependent manner (Fig. 4A). IC50 values at $24 \mathrm{~h}$ were $22.28 \mu \mathrm{M}$.

To further identify whether PI3K/AKT pathway was associated with PCPs cell proliferation,PCPs cells in logarithmic growth phase were treated with doses of 10, 20,30,60 and $90 \mu \mathrm{M}$ of LY294002, and cellular growth was measured by growth curve. The growth curve of tumor showed that LY294002 inhibited tumor cell growth(Fig. 4A). Then, a plate clone formation assay also showed that the clone number was markedly decreased by LY294002 treatment(Fig. 4B,C).

\section{LY294002 induced cell cycle arrest and apoptosis in PCPs cells}

PCPs cells in logarithmic growth phase were treated with doses of 10, 20, 30,60 and $90 \mu \mathrm{M}$ of LY294002, and cell cycle progression was determined using flow cytometry. The results of cell cycle detection showed that LY294002 treatments had an impact on the progression of the PCPs cell cycle $[$ for $24 \mathrm{~h}$ incubation time, LY294002 induced cell cycle arrest in PCPs cells at the G1 / M phase in dose dependent manner(Fig. 5B,D). And then the PCPs cells, after exposure to LY294002, were stained with Annexin V bound to FITC, and analyzed by flow cytometry to assess the apoptotic cell. Flow cytometry data corroborated the above findings that apoptosis was observed immediately in PCPs cells treated with LY294002 compared with untreated(Fig. 5A,C). Our data revealed that LY294002-treatment induced PCPs cells to undergo apoptosis.

\section{Effects of the PI3K inhibitor LY294002 on regulation of PCPs cell migration and invasion.}

We also determined the effects of PI3K inhibitor LY294002 on regulation of PCPs cell migration and invasion. The results of the Transwell tumor cell migration and invasion assays indicated that the migration and invasive ability of the PCPs cells decreased significantly following addition of LY294002(Fig. 6B,C). In scratch wound assay showed the cells of untreated controls have been migrated towards each other, with decreasing open wound area, while the motility of LY294002-treated PCPs cells was inhibited in scratch wound assay(Fig. 6A). 


\section{Discussion}

Craniopharyngioma represents one of the more difficult treatment paradigms in neurological tumor, and due to its low prevalence.Previous researches showed that mutations in CTNNB1 (encoding $\beta$-catenin), leading to the over-activation of the WNT pathway, underlie the molecular aetiology of human ACP[13].The molecular pathogenesis of PCP was largely unknown until exome sequencing studies identified BRAF mutations in 95\% (51/53) of these tumours but not in the ACP variant[15].It is well established that the gain-of-function mutation BRAF-V600E, a critical serine/threonine kinase in the RasRaf-Mek-ERK1/2 pathway, renders it a potent oncogene leading to increased cell proliferation and survival, resulting in cell transformation and tumorigenesis[16, 17].overexpression of PI3K induced cell transformation in a MAPK/Erk- and Akt/mTOR-dependent manner[18].Prior studies that have noted the importance of PI3K signalling within tumours. PI3K/Akt activation has been considered as a causative factor, aberrant activation of the PI3K/AKT pathway has been observed in a number of tumor tissues, including craniopharyngioma, and inhibitors of the PI3K/AKT pathway have been investigated as a potential anticancer therapy[19].In the present study, the protein expression of p-PI3K[p-AKT were upregulated in $\mathrm{CP}$ tumor tissue in comparison with normal brain tissue $(\mathrm{P}<0.001)$ (Fig. 1). $\mathrm{PI3K} / \mathrm{AKT}$ plays an important role in craniopharyngioma.

A number of study have show that PI3K/Akt inhibition may delay tumor growth,including renal cancer[20],breast cancer[21],glioblastoma[22], and neuroblastoma[23].In our study, The CCK-8 and plate colony formation assays demonstrated that LY294002 had a marked effect on PCP cell proliferation.LY294002 treatment significantly inhibited the proliferation potentials of PCP cell compared with the control group. Two mechanisms may explain the inhibitory effects of LY294002 on PCPs cancer cell proliferation. One is that LY294002 inhibits cell cycle progression, inducing specific G1 arrest, leading to an inhibition of cell proliferation. As demonstrated previously in human melanoma cells, LY294002 inhibits cell proliferation by inhibiting $\mathrm{G} 1$ cyclin-dependent kinase activity and the subsequent phosphorylation of retinoblastoma protein[24].The second possibility is that LY294002 increases apoptosis of PCPs, as recently suggested [24]. Ras was found to protect cells from apoptosis through activation of protein kinase B/Akt via PI3-K[25].LY294002, by inhibiting PI3-K activity, blocks the signal transduction pathway, which in turn may inhibit Ras-mediated protection from apoptosis in PCPs cancer.Studies have shown that LY294002 significantly inhibits the proliferation of PCPs cells,and increases apoptosis of PCPs cells.

in vitro studies, when PCPs cells were treated in vitro with the higher $(30 \mu \mathrm{m})$ dose of LY294002 for $24 \mathrm{~h}$, the effects appeared toxic. Cellularity was decreased, and the cell clusters appeared shrunken, with poor cellular cohesion. However, $24 \mathrm{~h}$ after withdrawal of $30 \mu \mathrm{m} \mathrm{LY} 294002$, there was partial recovery. The cellularity was decreased, but the cells regained cohesion, and their nuclei and cytoplasm more closely resembled the appearance of the untreated cells. These observations both in vitro imply that the side effects appearing in the LY294002-treated PCPs cells are largely dependent on the dose and the time course of the LY294002 treatment, both of which might be reduced to avoid these side effects. 
In summary, our data demonstrate that LY294002 markedly inhibits cancer cell proliferation in vitro, suggesting an important role of PI3K in the growth of PCPs and provide an attractive therapeutic target for developing a novel approach to treat PCPs.

\section{Declarations}

\section{Funding}

This work was supported by the National Natural Science Foundation of China (grant nos. 81560207 and 81460381), Ganpo555 engineering excellence of Jiangxi Science and Technology Department (2013), Key research and invention plan of Jiangxi Science and Technology Department (20192BBG70026), Natural Science Foundation of Jiangxi Province(2014ZBAB205019), Education Department of Jiangxi Province (GJJ13031)

\section{Availability of data and materials}

Data sharing is not applicable to this article, as no datasets were generated or analyzed during the current study.

\section{Authors' contributions}

LIN ZHOU and CHENXING OUYANG performed the experiments and generated data. LIN ZHOU, LINCHUN FANG SHIHAI LAN, XIAO WU囚YOUYUAN BAO SUYUE ZHENG SHAOYANG LI and MINDE LI analyzed data. HONG TAO designed experiments. TAO HONG wrote the manuscript. All authors reviewed and approved the manuscript.

\section{Ethics approval and consent to participate}

The present study was approved by the Research Ethics Committee of the First Affiliated Hospital, Nanchang University (Nanchang, China).

\section{Patient consent for publication}

Not applicable.

\section{Competing interests}

The authors declare that they have no competing interests.

\section{References}

1. Goschzik T, et al. Genomic Alterations of Adamantinomatous and Papillary Craniopharyngioma. J Neuropathol Exp Neurol. 2017;76(2):126-34. 
2. Preda V, et al. The Wnt signalling cascade and the adherens junction complex in craniopharyngioma tumorigenesis. Endocr Pathol. 2015;26(1):1-8.

3. Hölsken A, et al. Adamantinomatous and papillary craniopharyngiomas are characterized by distinct epigenomic as well as mutational and transcriptomic profiles. Acta Neuropathol Commun. 2016;4:20.

4. Brastianos $\mathrm{PK}$, et al. Exome sequencing identifies BRAF mutations in papillary craniopharyngiomas. Nat Genet. 2014;46(2):161-5.

5. Brown HA, Thomas PG, Lindsley CW. Targeting phospholipase $D$ in cancer, infection and neurodegenerative disorders. Nat Rev Drug Discov. 2017;16(5):351-67.

6. Martini M, et al. PI3K/AKT signaling pathway and cancer: an updated review. Ann Med. 2014;46(6):372-83.

7. Alzahrani AS. PI3K/Akt/mTOR inhibitors in cancer: At the bench and bedside. Semin Cancer Biol. 2019;59:125-32.

8. Gehringer F, et al. Physiological levels of the PTEN-PI3K-AKT axis activity are required for maintenance of Burkitt lymphoma. Leukemia. 2020;34(3):857-71.

9. Ho CJ, Gorski SM. Molecular Mechanisms Underlying Autophagy-Mediated Treatment Resistance in Cancer. Cancers (Basel), 2019. 11(11).

10. Wang $Y$, et al. Clinical and prognostic role of annexin A2 in adamantinomatous craniopharyngioma. J Neurooncol. 2017;131(1):21-9.

11. Nelson WG, et al. Specific keratins as molecular markers for neoplasms with a stratified epithelial origin. Cancer Res. 1984;44(4):1600-3.

12. Quentmeier $\mathrm{H}$, et al. Immunocytochemical analysis of cell lines derived from solid tumors. $J$ Histochem Cytochem. 2001;49(11):1369-78.

13. Zhang N. [Vimentin and tumor diagnosis]. Zhonghua Bing Li Xue Za Zhi. 1990;19(2):122-4.

14. Martinez-Barbera JP, Buslei R. Adamantinomatous craniopharyngioma: pathology, molecular genetics and mouse models. J Pediatr Endocrinol Metab. 2015;28(1-2):7-17.

15. Amit S, et al. Axin-mediated CKI phosphorylation of beta-catenin at Ser 45: a molecular switch for the Wnt pathway. Genes Dev. 2002;16(9):1066-76.

16. Davies $\mathrm{H}$, et al. Mutations of the BRAF gene in human cancer. Nature. 2002;417(6892):949-54.

17. Haston S, et al. MAPK pathway control of stem cell proliferation and differentiation in the embryonic pituitary provides insights into the pathogenesis of papillary craniopharyngioma. Development. 2017;144(12):2141-52.

18. Davis WJ, Lehmann PZ, Li W. Nuclear PI3K signaling in cell growth and tumorigenesis. Front Cell Dev Biol. 2015;3:24.

19. Shingu T, et al. Synergistic augmentation of antimicrotubule agent-induced cytotoxicity by a phosphoinositide 3-kinase inhibitor in human malignant glioma cells. Cancer Res. 2003;63(14):4044-7. 
20. Sourbier $C$, et al. The phosphoinositide 3-kinase/Akt pathway: a new target in human renal cell carcinoma therapy. Cancer Res. 2006;66(10):5130-42.

21. Mallon R, et al. Antitumor efficacy of PKI-587, a highly potent dual PI3K/mTOR kinase inhibitor. Clin Cancer Res. 2011;17(10):3193-203.

22. Zhao HF, et al. Recent advances in the use of PI3K inhibitors for glioblastoma multiforme: current preclinical and clinical development. Mol Cancer. 2017;16(1):100.

23. Mohlin S, et al. Anti-tumor effects of PIM/PI3K/mTOR triple kinase inhibitor IBL-302 in neuroblastoma. EMBO Mol Med. 2019;11(8):e10058.

24. Shayesteh L, et al. PIK3CA is implicated as an oncogene in ovarian cancer. Nat Genet. 1999;21(1):99-102.

25. Downward J. Ras signalling and apoptosis. Curr Opin Genet Dev. 1998;8(1):49-54.

\title{
Figures
}

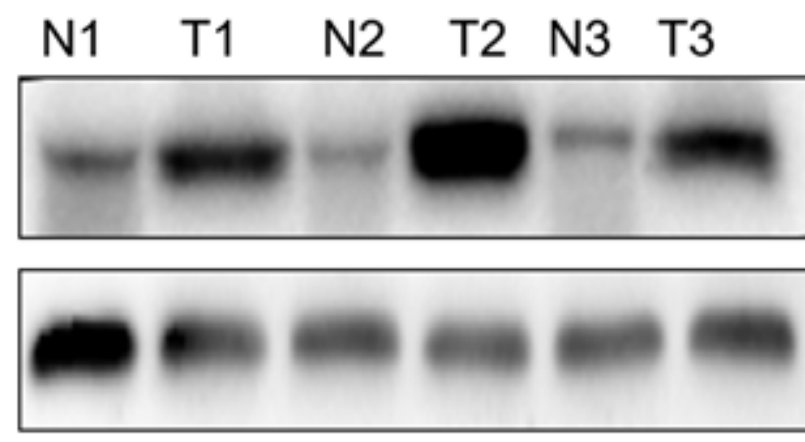

\author{
p-PI3K
}

PI3K

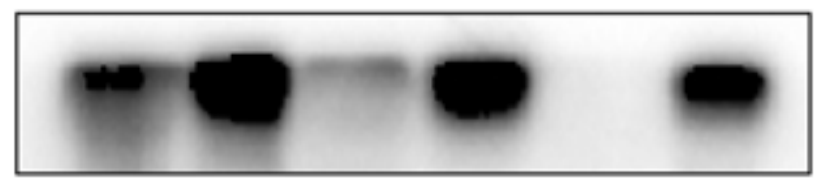

p-AKT

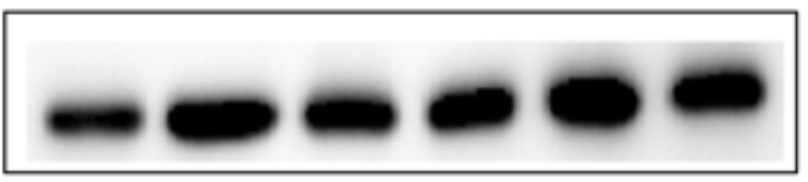

\section{AKT}

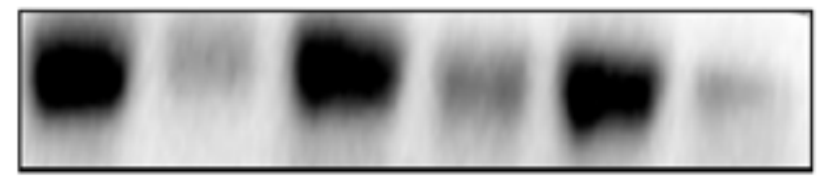

\section{caspase3}

\section{GAPDH}

\section{Figure 1}

The relative expression quantities of p-PI3K $\square$ p-AKT in craniopharyngioma tissues compared with normal controls were detected by western blotting. 


\section{vimentin \\ DAPI \\ Merge}
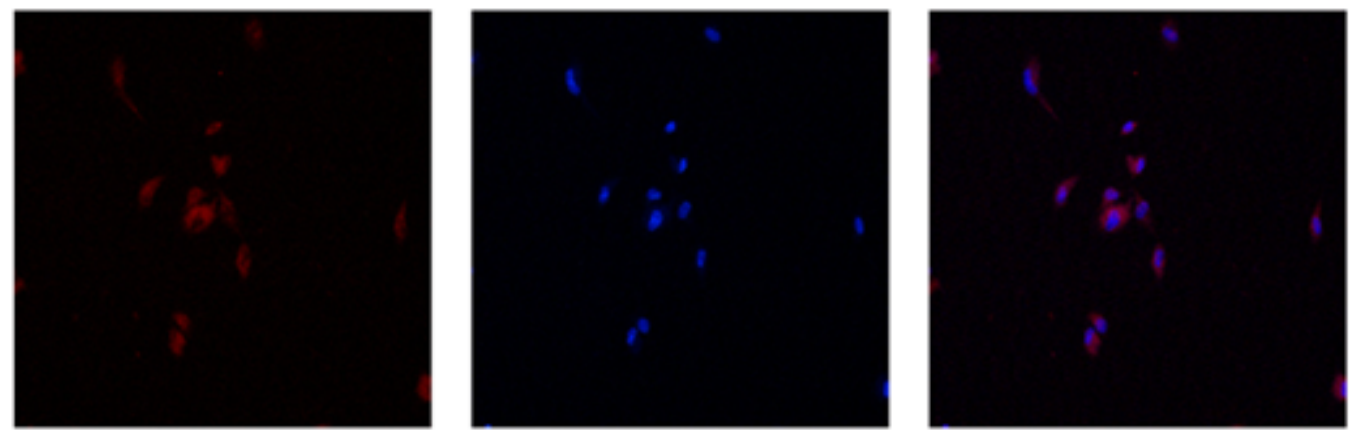

\section{keratin}

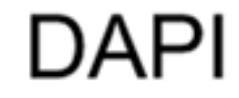

\section{Merge}
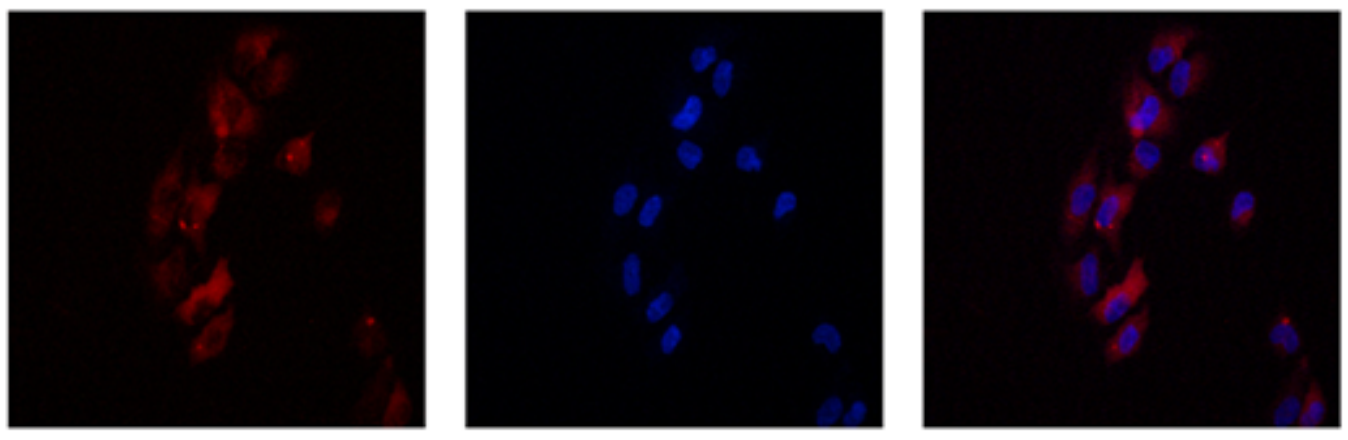

\section{Figure 2}

Validation of primary PCPs cells.Immunofluorescence of primary PCPs cell to detect pan-CK and VIM using DAPI to label DNA. PCPs, papillary craniopharyngioma; DAPI, 4',6-diamidino-2-phenylindole; panCK, pan cytokeratin; VIM, vimentin. 
A

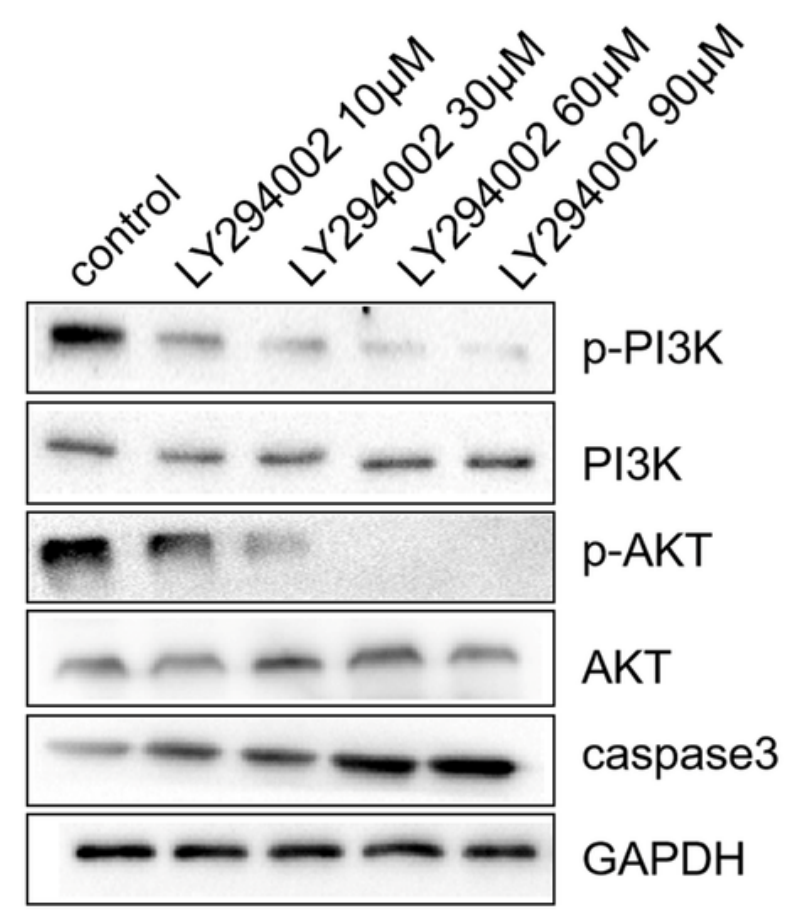

B
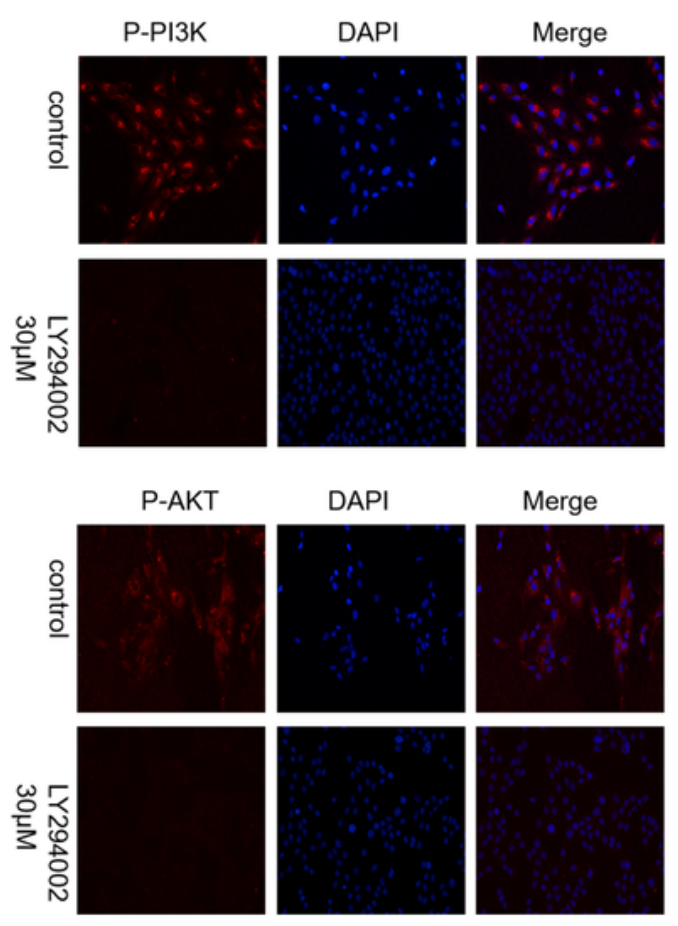

Figure 3

LY294002 influence p-PI3K $\square$ p-AKT expression in PCPs cells. Cells were treated with 30 $\mu$ m LY294002 for 24h,immounofluoence assay and WB was used to detect the expression of p-PI3K $\square p-A K T(A, B)$. 


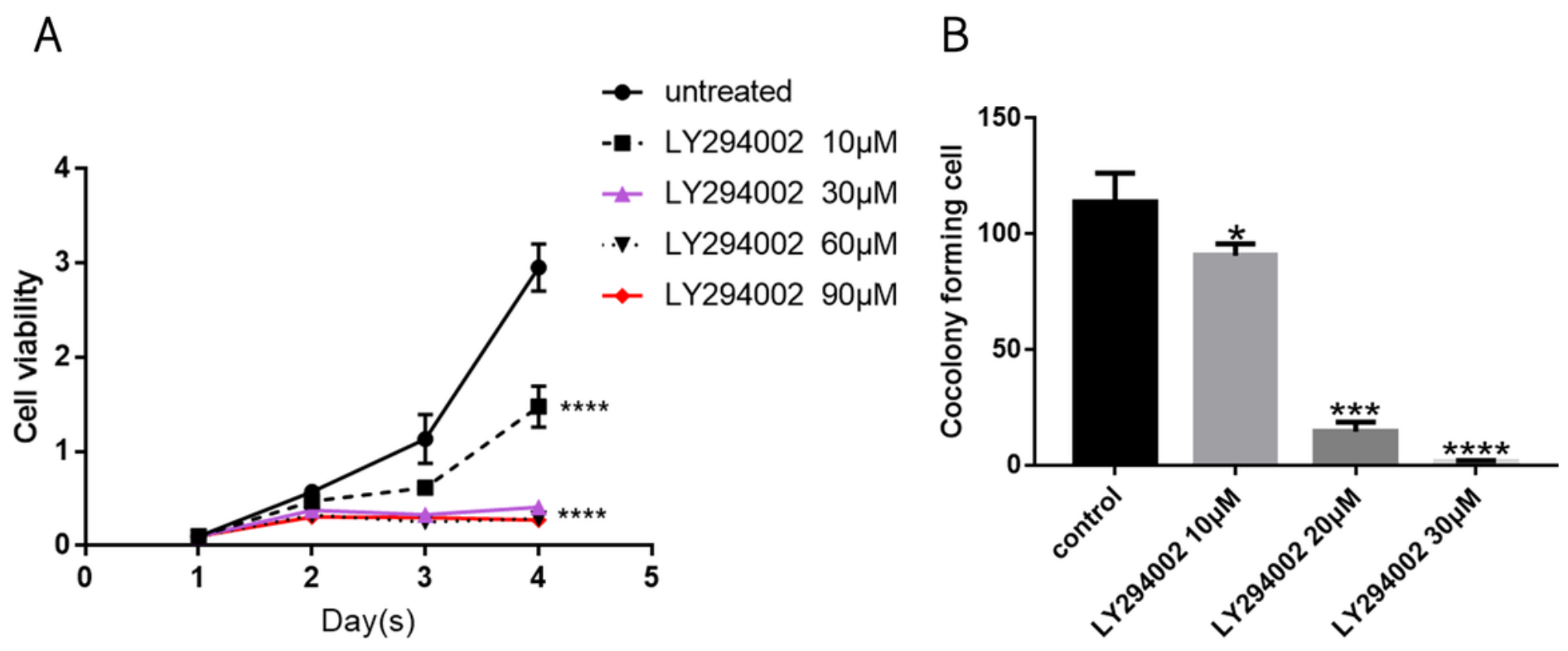

C

control

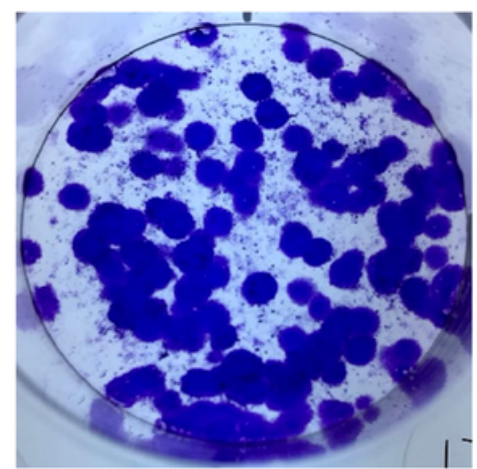

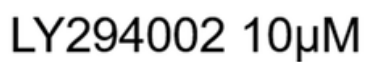

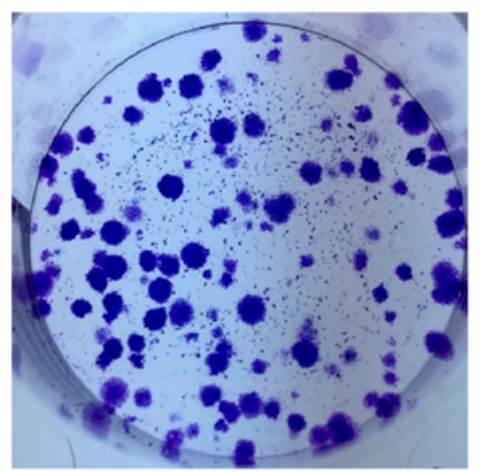

LY294002 20HM

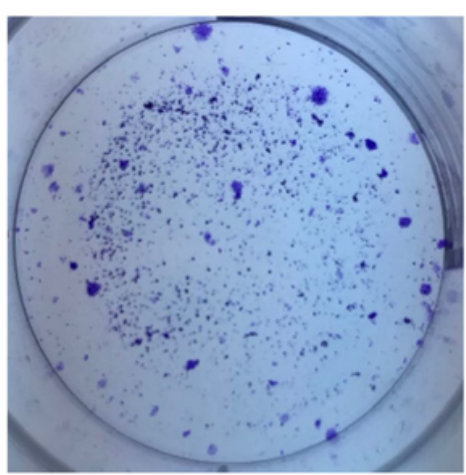

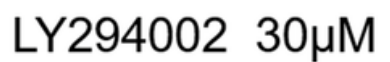

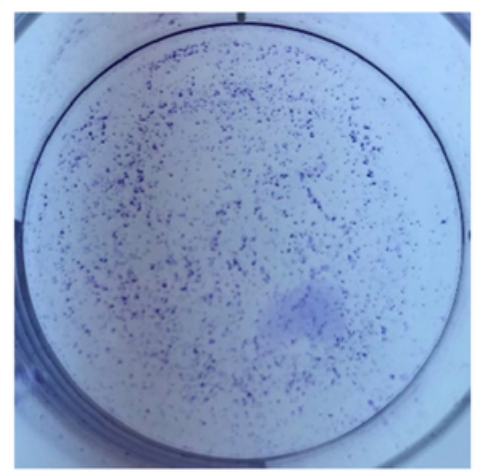

Figure 4

The influence of LY294002 on the proliferation of PCPs cells.CCK-8 ,colony formation assay detected that cell proliferation vitality $(A B) .(* \star \star p<0.001 ; * \star p<0.01 ; * p<0.05)$. 

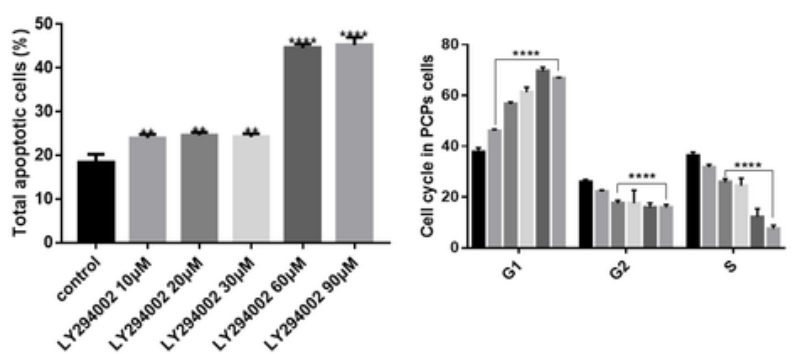

- control

- LY294002 10بM

- LY294002 20uM

- LY294002 30بM

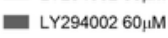

- LY294002 90رM
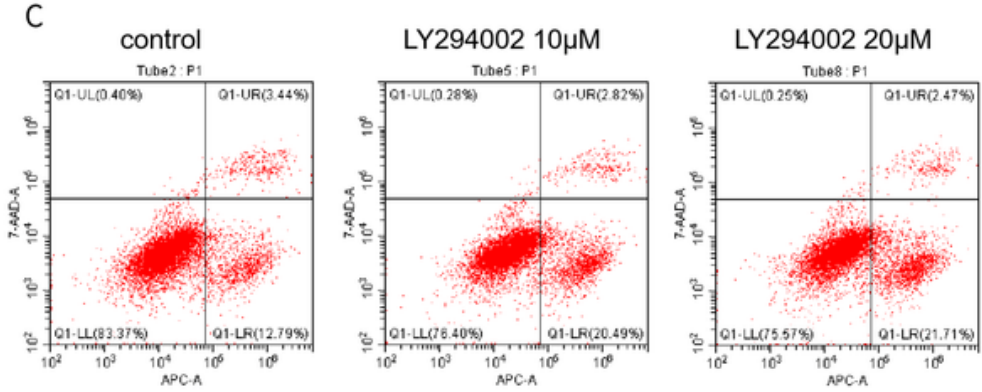

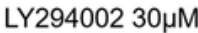

LY294002 60بM

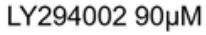
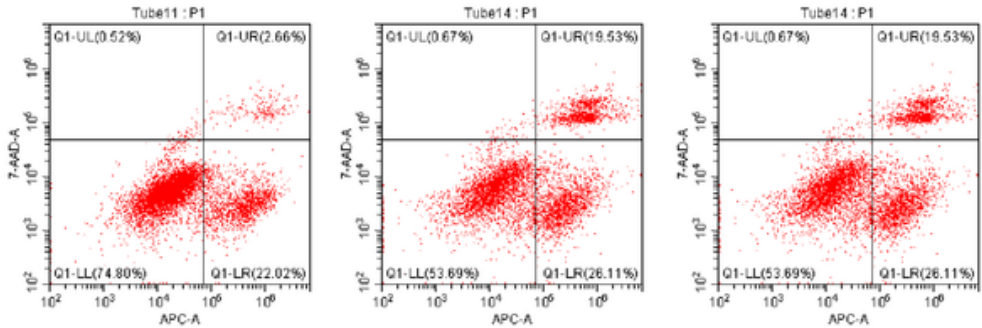

D

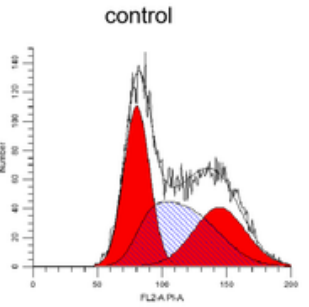

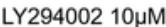

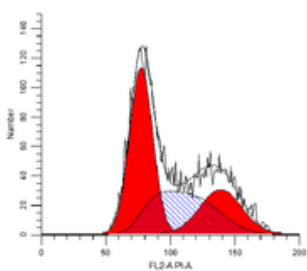

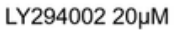

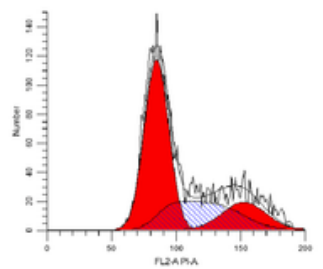

LY294002 30بM

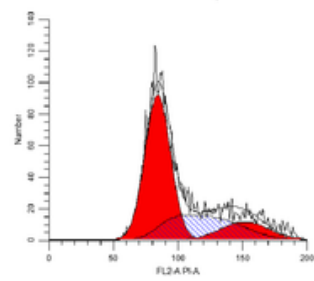

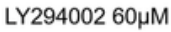

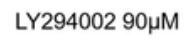
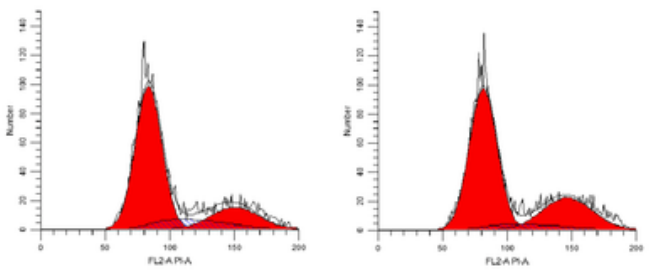

Figure 5

Effect of LY294002 on the apoptosis and cell cycle of PCPs cells. Cells were treated with or without 10, 20,30,60, and $90 \mu \mathrm{M}$ LY294002 for $24 \mathrm{~h}$. Apoptotic cell death was measured by Annexin V staining assay $(A, C)$.Cell cycle changes for PCPs cells(D), treated in increasing concentrations of the inhibitor displayed as bar graphs of different phases of the cell cycle (B) measured. 
A

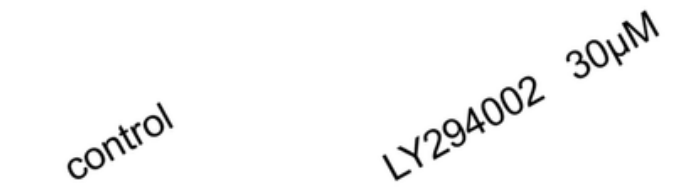
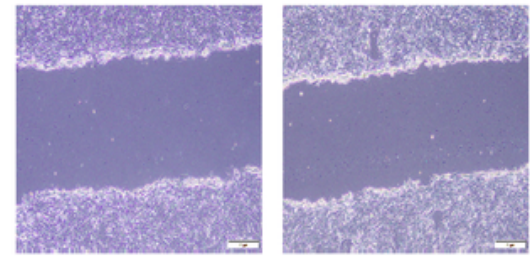

$6 h$
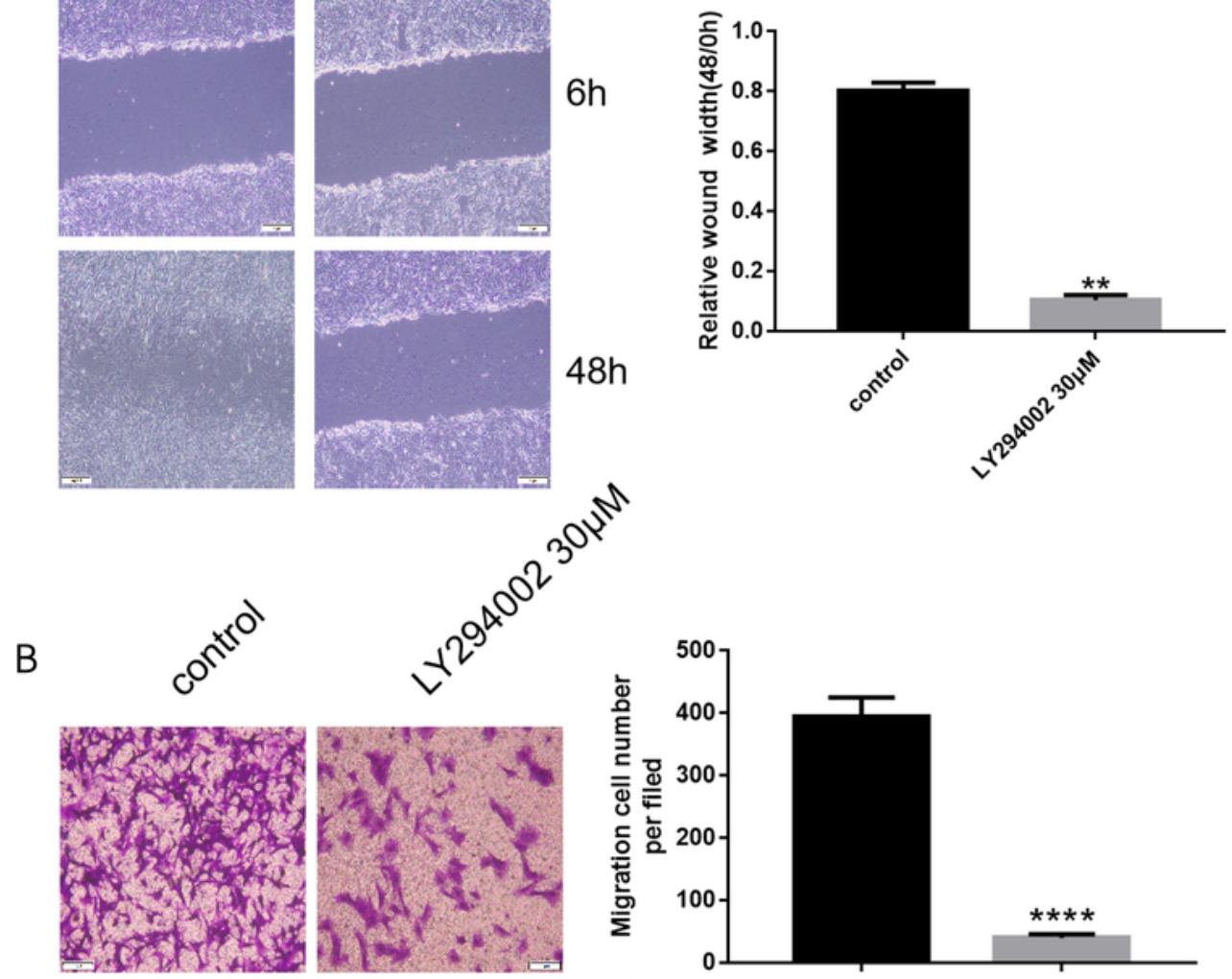<smiles>[AlH2]</smiles>

$48 \mathrm{~h}$

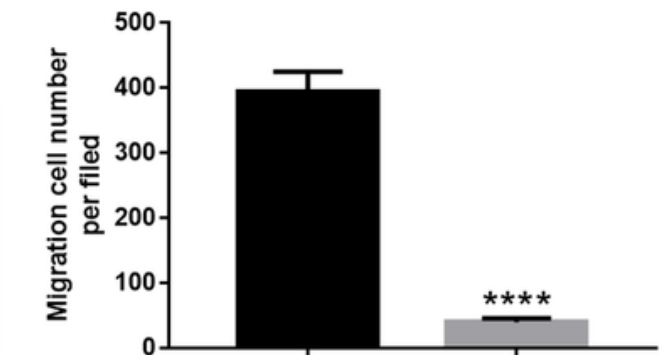

C
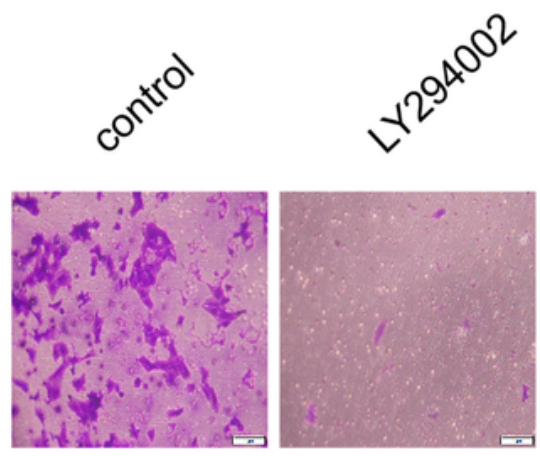<smiles>[CH][CH]</smiles><smiles>C1CCCC1</smiles>
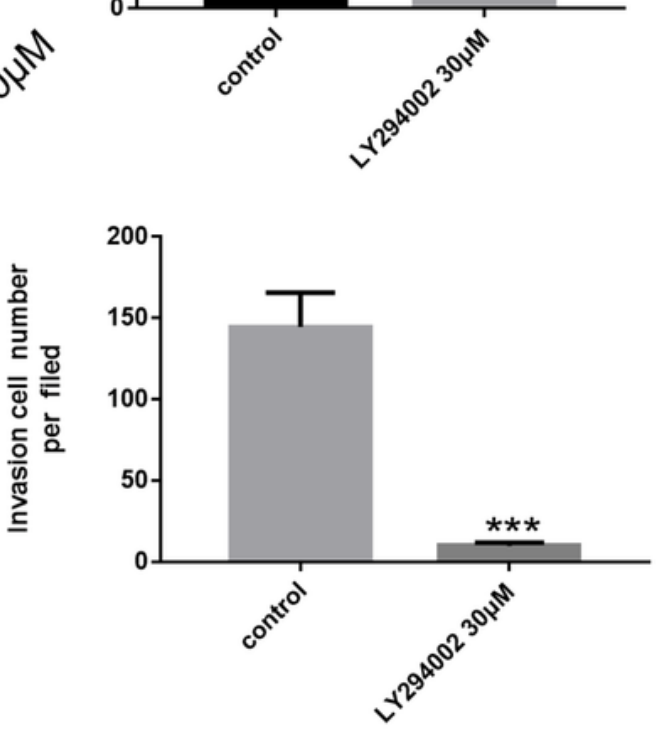

\section{Figure 6}

The influence of LY294002 on the migration and invasion of PCPs cells.Cells were treated with 30um LY294002 for24 h,wound healing \transwell assay was used to detect the changes of PCPs cells

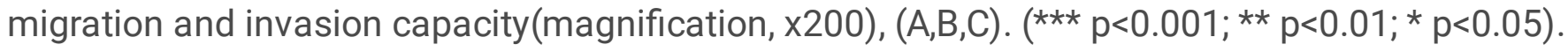

